\title{
The Study on Psychological Capital Development of Intrapreneurial Team
}

\author{
Zhenguo Zhao \\ School of Business, Tianjin Polytechnic University \\ Tianjin 300387, China \\ E-mail: xixiang123456@yahoo.com.cn \\ Jialin Hou \\ School of Business, Tianjin Polytechnic University \\ Tianjin 300387, China \\ E-mail: hj1629@163.com
}

\begin{abstract}
Intrapreneurship is an important way for enterprises to maintain the sustained growth. Furthermore, intrapreneurial team is a dominant force to achieve intrapreneurship, among which psychological capital (PsyCap) plays an important role. Therefore, the development of psychological capital of intrapreneurial team bears an important significance. Based on the study of related literatures, this paper presents a number of recommendations on the development of intrapreneurial teams' PsyCap.
\end{abstract}

Keywords: Intrapreneurial team, Psychological capital (PsyCap), Development

\section{Introduction}

With the advent of global competition and the intense changes in the external environment, for businesses to survive and develop, in particular to maintain the continued growth, enterprises must maintain the entrepreneurial spirit and conduct intrapreneurship. Intrapreneurial team is a fundamental guarantee for the successful implementation of intrapreneurship. When faced with setbacks or failures, intrapreneurial teams keep optimistic in most cases. First of all, they do not admit that they are defeated, regard failures as a temporary setback, learn from them and resolve them. Failure is not a reason for retreat. Second, they think they want to be responsible for their own destiny. Through dealing with their mistakes and failures in an objective way, the intrapreneurial teams have learned how to avoid repeating the same mistakes, which in turn helps them to achieve further success.

In accordance with the viewpoints of Luthans, Youssef, and Avolio, PsyCap refers to "the positive psychological development states of individuals." Intrapreneurial teams have a high level of self-efficacy, keep optimistic in the process of enterprise development and harbor a high level of hope, show the relatively strong resilience in the process of achieving team goals, which will certainly drive the intrapreneurial team to steadily enhance learning some kind of specialized technical knowledge and skills needed by enterprises, take the initiative to master the information useful for enterprises, and self-consciously understand and acquire the essence of the technical knowledge and skills through practice, combine knowledge, information and wisdom together, so as to promote enterprise-wide human capital level and boost enterprise's technological capabilities.

This shows that PsyCap is of great significance to intrapreneurial teams, and is an important factor for intrapreneurial teams to achieve success. Consequently, the development of intrapreneurial teams' PsyCap bears a great significance.

\section{Connotation of Psychological Capital}

In 2004, with the ideas of positive psychology and positive organizational behavior as the framework for reflection, Luthans et al. set forth the concept of "positive psychological capital" with emphasis on positive powers of individuals as the core following the analysis of characteristics and differences of economic, human and social capital, which makes scholars start to pay attention to the study on and its impact on leaders and employees. Luthans et al. pointed out that the PsyCap refers to the individuals' positive state of mind, which mainly includes the four aspects of confidence or self-efficacy, hope, optimism and resilience. What is different from the past understanding of the PsyCap is that they 
stressed the psychological capital can be measured, infinitely developed, and managed, while at the same time pointing out specific methods related to PsyCap investment, development and management.

In 2005, for the first time Luthans et al. explicitly defined psychological capital as "the core psychological elements of individuals' general positive nature, which is specifically represented as the state of mind to comply with the standards of positive organization performance. It is beyond human and social capital, and is able to make individuals obtain competitive advantages through the targeted input and development. And they pointed out that the definition can be understood from the following four aspects: First, it is based on the positive psychology paradigm (emphasizing the positivity and human advantages); Second, it is formed up of the psychological state to comply with the standards of positive organization conducts (such as uniqueness, theoretical and research base available, being effectively measured, and being represented as the state); third, it is beyond human capital (what you know, such as knowledge, skills, perspectives and capabilities) and social capital (who you know, such as trust, relationships, working networks connected with each other and friends), and it also concerns who you are (e.g. self-confidence, hope, optimism and resilience); Fourth, it improves performance and enhances the competitive advantage through its investment and development.

In 2007, Luthans, Yousseff and Avolio made a revision of the definition of psychological capital, thinking that the PsyCap refers to "an individual state of positive psychological development, which is characterized by: (1) possession of confidence (self-efficacy) by which challenging tasks can be successfully completed through showing and paying necessary efforts; (2) positive attribution should be made on the current and future success (optimism); (3) targets need to be adhered to, and if necessary the line of achieving goals can be re-selected in order to be successful (hope); (4) When confronted with the problems and difficulties, success can be achieved by sticking to goals, quickly restoring and taking roundabout ways "

Based on the above-mentioned analysis, PsyCap refers to positive psychological resources owned by an individual, and is useful in predicting a psychological state synthesis of individual high-performance work and happiness index. These positive mental states can lead to positive organizational behavior, make individuals diligently do the right thing and obtain higher performance and job satisfaction. Its components (confidence or self-efficacy, hope, optimism and resilience) are the state-like positive psychological forces, rather than tendentious, relatively stable and trait-like individual characteristics (such as due diligence or self-esteem, etc.), and psychological capital, together with human and social capital, makes up the human resources, which is owned by an individual, that can be objectively measured, invested \& developed, and effectively managed.

\section{Constituent Elements of PsyCap}

According to the concept of psychological capital, its constituent elements must have the following characteristics: stateful, developmental, enthusiastic, discriminant validity different from other concepts, and an impact on individual performance. Thus, confidence, hope, optimism and perseverance are considered PsyCap dimensions most suitable for the aforementioned criteria (Luthans, 2004). First of all, they can be developed, and are a positive state of mind that has an impact on employee performance. Speaking of hope, Snyder (2002) believed that it was composed of the will power, objectives and ways to achieve them, and that there are convergent validity and discriminant validity between hope and other positive states, but hope also has an important influence on managers and their staff's personal performance; the study of Larson et al (2007) also shows that hope has a positive effect on staff satisfaction, organizational commitment and work happiness, and there is discriminant validity compared with other concepts. As a result, hope is in line with the requirements of PsyCap concept, so it could be a dimension of psychological capital. The relevant confidence (self-efficacy) study is the most solid. Many studies have shown that the concept of confidence, which is stateful, developmental, and enthusiastic, is an independent concept, and is closely linked with staff's job performance. Optimism is a positive experience for the future. Peterson (2000) pointed out that the real optimism, which can overcome barriers on the road to success, can be a state that can be changed. Seligman (1998) also found that optimism had a major impact on the work efficiency of insurance staff. Youseff (2006) also pointed out that optimism is highly correlated with employee satisfaction, work happiness, and work performance. Similarly, a large number of studies (Luthans, 2005; Larson 2006) have also proved that resilience can be an excellent personal potential that can be cultivated and changed, and have an important impact on the performance of employees. In addition, there is discriminant validity based on empirical analysis in the above-mentioned four factors. (Magaletta \& Oliver, 1999; Youssef \& Luthans, 1998). Meanwhile, there is a factor that plays a common role in the deeper level of these concepts. It closely combines them together and is probably the high-level factor_— PsyCap. Hence, this paper has every reason to believe that PsyCap is made up of confidence, hope, optimism, and resilience, and that it represents the variance shared between these four variables.

In this article, self-efficacy (or confidence) refers to the confidence level of the role that individuals play of their motivation, cognitive resources and action steps, and of the ability of the specific tasks in a given scenario of successful management; hope means a positive motivational state of the sense of success resulting from the interaction based on 
between agent (goal-oriented activity) and the path ( plan to achieve its objectives); optimism can be defined by the two key dimension (persistence and pervasiveness) of the people's explanatory style about good and bad events to define, and refers to the positive explanatory style by which a good case is ascribed to the internal, lasting and pervasive reason, and the bad events attributed to external and temporary reason under the specific scenario; resilience is an ability to recover quickly from adversity. People with this kind of resilience are characterized by steadily accepting the reality, firmly believing the life meaning under the support of stable values, and having the capability of supernaturally coping with and adapting to big changes on a temporary basis.

\section{Development of Intrapreneurial Teams' PsyCap}

Intrapreneurial team is a kind of team requiring strong adaptability and stress resistance, and its goal is to support fulfilling tasks in the environment in a highly efficient way and to satisfy stakeholders. Therefore, the key to improving the efficiency of task execution is to ensure that every member of the team are aware of and support overall and individual goals, thus making a personal contribution to the team as much as possible. PsyCap is an individual positive state of mind, and it provides for team members a better psychological understanding and transformations, thereby helping them take the initiative to understand and support team goals and finally urge themselves to do their utmost to complete tasks. If team members have a positive psychological capital, then individual psychological expectations and team goals are easier to reach a consensus and form a positive psychological contract, so that their mental states can be adjusted in time even if there are deviations from the team target.

Because PsyCap is the sublimation and integration of resources and such positive psychological states as individual optimism, hope, confidence and resilience, and has great development potential, both intrapreneurial teams and individual members should consciously try to obtain, maintain and enhance it. Now, this paper proposes a few basic advices for intrapreneurial team members and teams from the following angles.

\subsection{Advice on PsyCap development of team members}

In 2005, Prof. Luthans proposed the famous psychological capital intervention (PCI) (For details, please refer to attached Fig. 1), and through empirical studies validated the model. As for fostering hope, cultivating the spirit of optimism, enhancing self-efficacy (confidence) and strengthening the resilience force, the model put forward a set of highly operational facilitation measures, thus offering a good way of thinking for enterprise's practice of PsyCap intervention and development.

According to Prof. Luthans' PCI model, the following suggestions are raised on PsyCap development of team members:

\subsubsection{Development of hope}

Hope is determined by the two major factors, namely the path and willpower. Path refers to the method, strategy or ability to achieve goals. People with high hope usually effectively find or produce a variety of ways to achieve targets. Willpower means the motive or belief to pursue goals, and is the psychological energy of achieving objectives. This energy can encourage individuals to move forward continuously, that is to say that when individuals encounter setbacks, they persist in the commitment to solve the problem through faith, thus leaving them equipped with the courage and confidence to achieve their goals. The basic principle of hope development is to strengthen the training objectives, motivation and means, and focuses on the obvious role communication. It can be specifically conducted through the following ways: First, specific, challenging and measurable team and personal goals need to be identified. It should be broken down into the stage sub-goals easy to manage and realize, which would make it easier to reach and access to stage success and the direct experience of success. Secondly, power to achieve hope is enhanced through employee involvement and empowerment. Participation and empowerment can enhance the feelings about staff grasping themselves and the team's present and future development, and when leaders are showing the confidence in employees and considering them as the talent who would be successful, the motivation and willpower expected to achieve can be substantially increased. Third, a number of alternative ways to continuously reach goals need to be figured out, and corresponding course of action worked out to boost the "readiness". Inner drill can be used to image important upcoming events, anticipate potential obstacles, make an assuming analysis of an accident, and to explore the choice of responding to plans, thereby enhancing readiness to deal with obstacles. In a volatile era, ways of choice need to be expanded to make it more open and efficient. Fourth, the willpower ought to be enhanced, and a preliminary framework for understanding of reality can be formed through clear and obvious ways. Even if obstacles arise, the staying power to overcome will be motivated and giving up will not be chosen because of temporary difficulties. Fifth, staff needs to be encouraged to enjoy pleasure gained in the process of accomplishing the goal, instead of being just concerned about the final result. Sixth, the skills in dealing with specific conditions should be equipped with. That is to say that when the original way to achieve objectives is not feasible or not effective, staff can determine to choose what time and which alternative ways to achieve the objectives. Program planning and training help in this kind of skill-building. Seventh, team members ought to know when and how to re-set the target in order to avoid sliding into a false trap of hope, when encountered with insurmountable obstacles. 


\subsubsection{Development of optimism}

The ideal type of optimism should be realistic and flexible. Realistic optimism would not take extreme measures to justify the disliking choice and shirk personal responsibility; flexible optimism allows people to use different methods of interpretation to regard optimism as a type of adaptation to their own situation. The success and positive encouragement of other employees among the team will have a positive impact on staff and encourage them to complete the goal in a more optimistic state of mind. In the team, the methods of optimism development include: first, "be tolerant of the past." Re-planning needs to be learned and past failures and setbacks accepted. Past mistakes should be forgiven so as to be no longer subject to setbacks. Second, "the present is correctly evaluated." Be thankful to and satisfied with the success of their current lives, including those they can control and can not. Third, the "opportunities need to be sought for the future." The future and things difficult to determine can be seen as opportunities for growth and improvement, and the future should be embraced in a positive, happy, and confident attitude.

\subsubsection{Development of self-efficacy}

In the team, methods of self-efficacy development are mainly as follows: first, staff training intervention mode. Self-efficacy theory suggests that effective training technology is a staff training intervention mode comprised of four basic stages: (1) behavior and actions explained and demonstrated by examples; (2) learning throng discussion (LTD); (3) effective feedback approach; (4) re-training transfer. Second, feedback information and program management, which is in the process of performance evaluation, need to be perfected. Self-efficacy is an important intermediary variable affecting job performance. Studies found that positive feedback will increase the staff's self-efficacy, and negative feedback lead to a decline in self-efficacy. Third, approaches of training team members on self-regulating when faced with setbacks and occupational stress. (1) Emotion regulation. In overcoming the difficulties and actively responding to setbacks, positive emotions will maintain the original motives and expectations for future success, thus actively striving to maintain positive response. (2) Summation and reflection of the scheme. Work ought to be conducted according to plan, which determines a person's ability to effectively use time and energy and sum up lessons in order to benefit future battles. (3) The regulation of the will. Team members need to set goals, be undaunted by repeated setbacks, and believe that they can grapple with potential threats.

\subsubsection{Development of resilience}

Resilience allows individual and environmental protection mechanisms to improve its protection skills by increasing benefits and reducing risk factors. Three cognitive factors of resilience are: tolerance of the fact; firm convictions, and obtaining strength from the firm values; possession of the incredible capabilities to prepare for and adapt to significant changes at any time. The resilience development of team members can be implemented in three ways: First, it can be regulated through emotion. It is conducive to positive emotions of mental and physical health, can increase the power, mobilize staff potential, and enhance the ability to adapt to the environment. Second, it is regulated through language. Appropriate language can be used to eliminate employee' mental and physical displeasure, while the use of inappropriate language will increase both physical and mental frustration of the staff, leading to a serious illness. Third, it is regulated through study. Individual behavior is learned from the social environment, and abnormal psychology and behavior are a result of wrong and undesirable social pressures. Accordingly, study can also be used to eliminate stress and enhance resilience.

\subsection{Proposals on PsyCap development of intrapreneurial teams}

\subsubsection{To create a harmonious team environment}

Favorable team environment is a supportive environment of team externally and internally, and it is the fertile ground for cultivating positive PsyCap. In such circumstance, team members will take the initiative to upgrade their PsyCap, and the team will be investing heavily in the development of members' PsyCap, thus promoting the positive exertion of employees' PsyCap. This supportive team environment featuring benign interaction will play a positive role in the development of intrapreneurial teams' PsyCap.

\subsubsection{To establish an effective, interactive team culture}

Team culture is a deep identification with value. Encouragement of communication and information interaction is the basic operation mechanism of intrapreneurial teams. Under this mechanism, members' mind will be open, and team members take the initiative to upgrade their psychological capital. The team culture characterized by interactive learning and information-sharing is the essential culture connotation of intrapreneurial teams, as well as a culture guarantee of upgrading intrapreneurial teams' PsyCap.

\subsubsection{To offer different strategies for different individual characteristics}

Intrapreneurial team members have psychological diversity and dynamics, along with distinctive personal characteristics. Each person's psychological maturity and expectations of value vary from person to person. Therefore, if different strategy support and development are given to different employees with a definite goal in mind, then the 
individual members will feel the warmth of the team, and their sense of happiness will increase, which is not only conducive to bring into play and realize individual value, but also better suited to the cultivation and development of individual PsyCap.

4.2.4 To cultivate PsyCap from the perspective of strategic human resource management

Viewing the human resource management of intrapreneurial teams from a strategic level, it should be noted that there is a consistency between human resource management practices and the team targets. To build intrapreneurial teams, it should be noted that intrapreneurial teams' PsyCap is developed and its values brought into play through the flexible work setting, widespread participation, stable employment, goal-oriented performance management, incentive pay policy and other strategic measures.

4.2.5 To establish and improve employee' PsyCap by encouraging employees to learn and improve their practical ability to adapt to changes in the team

Intrapreneurial teams particularly emphasize on the upgrade of staff's learning ability and adaptability, aimed to train staff on adjusting to the need for change in the team. Because intrapreneurial teams are always working in a dynamic environment and demand constant changes, therefore, prior to the changes in the team, members should be encouraged to learn how to enhance the value of PsyCap and how to increase its own occupational safety, so as to meet the new changes and challenges.

4.2.6 To offer the guarantee to PsyCap fostering and development from the system level

PsyCap intervention and development of staff require a certain degree of institutional guarantee, but PsyCap development system under the institutional guarantee is a premise for successful intervention and development of PsyCap. To build PsyCap development system from the employee - team's point of view is to combine the teams' and individual PsyCap management strategies and ensure the consistency and effectiveness of PsyCap intervention and development measures from the system angle.

\section{References}

Luthans F, Youssef C M, \& Avolio B. J. (2007). Psychological capital: Developing the human competitive edge .Oxford, UK: Oxford University Press.

Luthans, F. (2005).Organizational behavior. New York: McGraw- Hill.

Seligman M E P. (2002). Authentic happiness .New York: Free Press. 


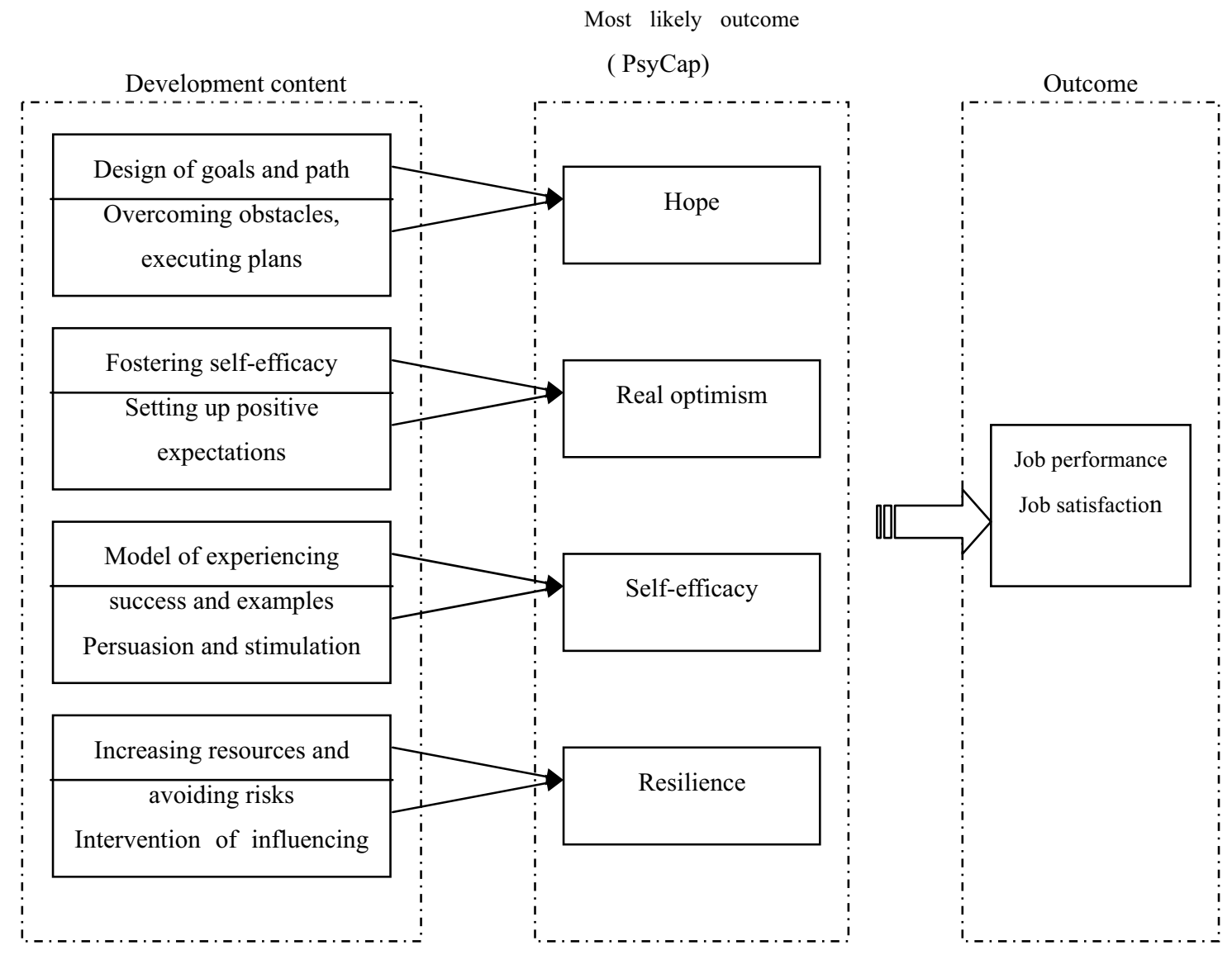

Figure 1. Influencing effects of PCI 